\title{
Operations on Dombi Bipolar Fuzzy Graphs Using T-Operator
}

\author{
R. Jahir Hussain and S. Satham Hussain
}

\begin{abstract}
-
\end{abstract}
\section{Index Terms-}

The bipolar fuzzy models give more accurate precision, flexibility and compatibility to the system when compared to the classical and fuzzy models. In 1994, Zhang [11], [12] introduced the concept of bipolar fuzzy sets as a generalization of fuzzy sets. Well known that, bipolar fuzzy sets are an extension of fuzzy sets whose membership degree range is $[-1,1]$. In a bipolar fuzzy set, the membership degree of an element means that the element is irrelevant to the corresponding property, the membership degree $(0,1]$ of an element indicates that the element somewhat satisfies the property, and the membership degree $[-1,0)$ of an element indicates that the element which satisfies the implicit counter property. In many fields, it is crucial to deal with bipolar information. It is stressed that positive information represents what is granted to be possible, where as negative information represents what is considered to be impossible. This domain has recently motivated in literature in several directions. For instance, consider the spatial relations: Human beings consider left and right as opposite directions. But this does not mean that one of them is the negation of the other. The semantics of opposite captures a notion of symmetry rather than a strict complementation. In particular, there may be positions which are considered neither to the right nor to the left of some reference object, thus leaving some room for indetermination. This corresponds to the idea that the union of positive and negative information does not cover the whole space. In 2011, Akram [1] introduced the notion of bipolar fuzzy graphs with various methods of their construction and also discussed the concept of isomorphism of these graphs. The bipolar fuzzy influence graph of a social group is described in [2]. Bipolar regular graphs and a result on an isomorphism between two bipolar fuzzy graphs follows from an isomorphism of their corresponding bipolar fuzzy line graphs are investigated in [3]. Recently, bipolar fuzzy graphs received much attention like bipolar fuzzy competition graphs are applied in politics see [7], [9], [10] and references therein. In [6], using the concept of path and strength of connectedness of bipolar fuzzy graphs, triple connected domination bipolar fuzzy graph is established. New operations on bipolar fuzzy graph namely direct product, semi strong product and strong product are studied in [8].

The operations of $\mathrm{t}$-norm and $\mathrm{t}$-conorm introduced by Dombi [5] is said to be Dombi operations which leads to good flexibility with the general parameter. The Dombi operations have so far not yet been applied for bipolar fuzzy graphs. In this paper, we defined some operations on Dombi bipolar fuzzy graphs. The goal of this paper is to mention that the $\max$ and $\min$ operations are not the only candidates for the generalization of the classical graphs to fuzzy graphs. We propose to incorporate the advancement proposed in [4], here we employed an operator, particularly T-operator, namely the Dombi operator in the area of bipolar fuzzy graph theory and the proposed concepts are provided with an appropriate examples.

In this paper, the concept of Dombi bipolar fuzzy graph is introduced and obtained that the direct product, join and ring sum of two Dombi bipolar fuzzy graphs are the Dombi bipolar fuzzy graphs. Generally, the Cartesian product and strong product of two Dombi bipolar fuzzy graphs are not Dombi bipolar fuzzy graphs. However, these graphs may be Dombi bipolar fuzzy if they have crisp vertices. Moreover, these results on Dombi bipolar fuzzy graphs are considered preserving strong property. In the real world, the Dombi bipolar fuzzy graph can be a description of the uncertainty of all kinds of networks. The theoretical results presented in this paper will improve the results mentioned in [4], and in future work, we investigate metric aspects of neutrosophic soft Dombi bipolar fuzzy graphs and the degree of the resultant graphs will also determined.

\section{Preliminaries}

The following definitions are used to prove the main results. Definition 1.1: [4] A binary operation $T:[0,1] \times[0,1] \rightarrow$ $[0,1]$ is a triangular norm (t-norm), if for every $u, v, w \in[0,1]$, which satisfies the following conditions:

(1) $T(1, u)=u$

(2) $T(u, v)=T(v, u)$ 
(3) $T(u, T(v, w))=T(T(u, v), w)$

(4) $T(u, v) \leq T(u, w)$ if $v \leq w$.

Definition 1.2: Possible choices for t-norms and dual tconorms are as follows:

\$ The minimum operator $M: M(u, v)=\min (u, v)$

A. The maximum operator $M^{*}: M^{*}(u, v)=\max (u, v)$

The product operator $P: P(u, v)=u v$

क The probabilistic operator $P^{*}: P^{*}(u, v)=u+v-u v$

The Lukasiewicz's t-norm $W: W(u, v)=\max (u+v-1,0)$

The bounded sum $W^{*}: W^{*}(u, v)=\min (u+v, 1)$.

We define the set of $T$-operator as

$$
T(u, v)=\frac{u v}{u+v-u v}
$$

which is obtained by taking $\lambda=0$ in Hamacher family and $\lambda=1$ in Dombi family of t-norms and t-conorms. Also it follows that, $P(u, v) \leq u v /(u+v-u v) \leq M(u, v), M^{*}(u, v) \leq$ $u v /(u+v-u v) \leq P^{*}(u, v)$.

Definition 1.3: Let $X$ be a non-empty set. Then the mapping $A=\left(\mu_{A}^{P}, \mu_{A}^{N}\right): X \times X \rightarrow[0,1] \times[-1,0]$ a bipolar fuzzy relation on $X$ such that $\mu_{A}^{P}(x y) \in[0,1]$ and $\mu_{A}^{N}(x y) \in[-1,0]$.

Definition 1.4: Let $A=\left(\mu_{A}^{P}, \mu_{A}^{N}\right)$ and $B=\left(\mu_{B}^{P}, \mu_{B}^{N}\right)$ be a bipolar set on $X$. If $A=\left(\mu_{A}^{P}, \mu_{A}^{N}\right)$ is a bipolar fuzzy relation on a set $X$ then $A=\left(\mu_{A}^{P}, \mu_{A}^{N}\right)$ is said to be a bipolar fuzzy relation on $B=\left(\mu_{B}^{P}, \mu_{B}^{N}\right)$. If $\mu_{B}^{P}(x, y) \leq \min \left(\mu_{B}^{P}(x), \mu_{B}^{P}(y)\right)$ and $\mu_{B}^{N}(x, y) \geq \max \left(\mu_{B}^{N}(x), \mu_{B}^{N}(y)\right)$ for every $x, y \in X$. A bipolar fuzzy relation $A$ on $X$ is symmetric if $\mu_{A}^{P}(x, y)=$ $\mu_{A}^{P}(y, x)$ and $\mu_{A}^{N}(x, y)=\mu_{A}^{N}(y, x)$ for every $X$.

\section{DOMBi Bipolar FuZzy GRAPHS}

Definition 2.1: A dombi bipolar fuzzy graph $G=(V, A, B)$ is a non-empty finite set on $V$ together with a pair of functions $A=\left(\mu_{A}^{P}, \mu_{A}^{N}\right): V \rightarrow[0,1] \times[-1,0]$ and $B=\left(\mu_{B}^{P}, \mu_{B}^{N}\right):$ $V \times V \rightarrow[0,1] \times[-1,0]$ such that for every $x, y \in V$,

$$
\begin{gathered}
B_{1}^{P}(x y) \leq \frac{A_{1}^{P}(x) A_{1}^{P}(y)}{A_{1}^{P}(x)+A_{1}^{P}(y)-A_{1}^{P}(x) A_{1}^{P}(y)} \quad \text { and } \\
\quad B_{1}^{N}(x y) \geq \frac{A_{1}^{N}(x) A_{1}^{N}(y)}{A_{1}^{N}(x)+A_{1}^{N}(y)-A_{1}^{N}(x) A_{1}^{N}(y)}
\end{gathered}
$$

Then one can say that $A=\left(A_{1}^{P}, A_{1}^{N}\right)$ as the dombi bipolar vertex set of $G$ and $B=\left(B_{1}^{P}, B_{1}^{N}\right)$ as the dombi bipolar edge set of $G$.

Example 2.1: Consider a dombi bipolar fuzzy graph $G$ such that $A=(a, b, c, d), B=\{(a b),(b c),(c d),(d a)\}$. By routine evaluation, we have

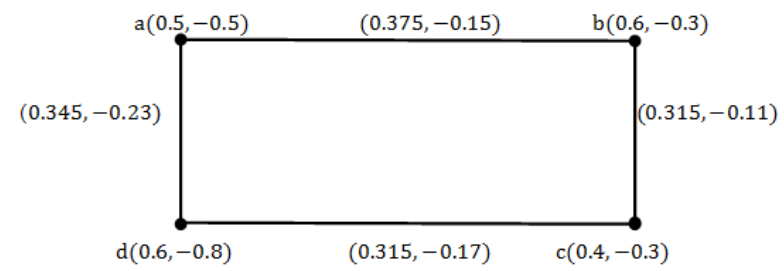

$\mathrm{G}$

Figure 1 Dombi Bipolar Fuzzy Graph
Definition 2.2: Let $A_{i}$ be the bipolar fuzzy subset of $V_{i}$ and let $B_{i}$ be the fuzzy subset of $E_{i}, i=1,2, \ldots$ Define the direct product of $\left(G_{1} \times G_{2}\right)=\left(A_{1} \times A_{2}, B_{1} \times B_{2}\right)$ of the dombi bipolar fuzzy graphs $G_{1}=\left(A_{1}, B_{1}\right)$ and $G_{2}=\left(A_{2}, B_{2}\right)$ respectively, given as follows:

(i) $\left(A_{1}^{P} \times A_{2}^{P}\right)\left(x_{1}, x_{2}\right)=\frac{A_{1}^{P}\left(x_{1}\right) A_{2}^{P}\left(x_{2}\right)}{A_{1}^{P}\left(x_{1}\right)+A_{2}^{P}\left(x_{2}\right)-A_{1}^{P}\left(x_{1}\right) A_{2}^{P}\left(x_{2}\right)}$ for every $\left(x_{1}, x_{2}\right) \in V_{1} \times V_{2}$, and

$\left(A_{1}^{N} \times A_{2}^{N}\right)\left(x_{1}, x_{2}\right)=\frac{A_{1}^{N}\left(x_{1}\right) A_{2}^{N}\left(x_{2}\right)}{A_{1}^{N}\left(x_{1}\right)+A_{2}^{N}\left(x_{2}\right)-A_{1}^{N}\left(x_{1}\right) A_{2}^{N}\left(x_{2}\right)}$ for every $\left(x_{1}, x_{2}\right) \in V_{1} \times V_{2}$

(ii)

$$
\begin{gathered}
\left(B_{1}^{P} \times B_{2}^{P}\right)\left(\left(x_{1} x_{2}\right),\left(y_{1}, y_{2}\right)\right) \\
B_{1}^{P}\left(x_{1} y_{1}\right) B_{2}^{P}\left(x_{2} y_{2}\right) \\
B_{1}^{P}\left(x_{1} y_{1}\right)+B_{2}^{P}\left(x_{2} y_{2}\right)-B_{1}^{P}\left(x_{1} y_{1}\right) B_{2}^{P}\left(x_{2} y_{2}\right)
\end{gathered}
$$

for every $\left(x_{1} x_{2}, y_{1} y_{2}\right) \in E_{1} \times E_{2}$ and

$$
\begin{gathered}
\left(B_{1}^{N} \times B_{2}^{N}\right)\left(\left(x_{1} x_{2}\right),\left(y_{1}, y_{2}\right)\right) \\
=\frac{B_{1}^{N}\left(x_{1} y_{1}\right) B_{2}^{N}\left(x_{2} y_{2}\right)}{B_{1}^{N}\left(x_{1} y_{1}\right)+B_{2}^{N}\left(x_{2} y_{2}\right)-B_{1}^{N}\left(x_{1} y_{1}\right) B_{2}^{N}\left(x_{2} y_{2}\right)}
\end{gathered}
$$

for every $\left(x_{1} x_{2}, y_{1} y_{2}\right) \in E_{1} \times E_{2}$

Example 2.2: Consider two dombi bipolar fuzzy graph $G_{1}$ and $G_{2}$, where $G_{1}=\left(A_{1}, B_{1}\right)$ $G_{1}=\{a(0.5,-0.6), b(0.4,-0.3)\}, \quad G_{2}=$ $\{c(0.7,-0,4), d(0.5,-0.4), e(0.6,-0.5)\}$

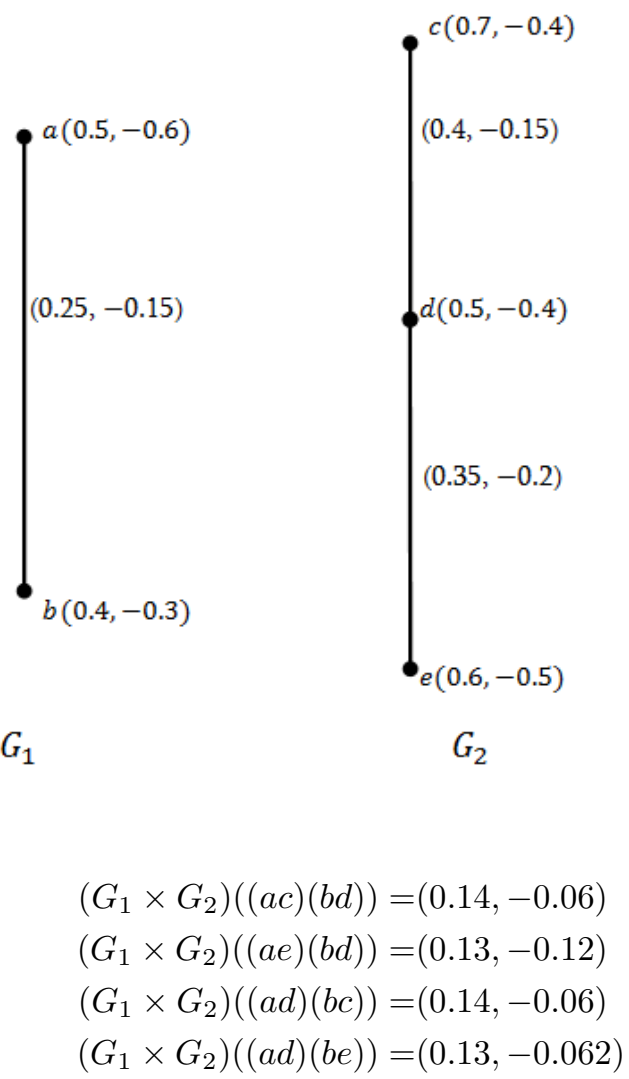

Hence it is easy to verify that $G_{1} \times G_{2}$ is the dombi bipolar graph of $G_{1}$ and $G_{2}$. 


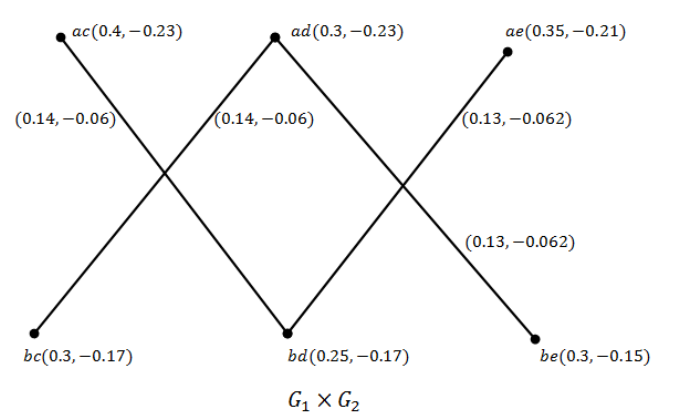

Figure 2 Direct product of Dombi Bipolar Fuzzy graph

Proposition 2.3: Let $G_{1}$ and $G_{2}$ be the dombi bipolar fuzzy graphs then the direct product $G_{1} \times G_{2}$ of $G_{1}$ and $G_{2}$ is the dombi bipolar fuzzy graph.

Proof: Consider $x_{1} y_{1} \in E_{1}, x_{2} y_{2} \in E_{2}$. Then

$$
\begin{aligned}
& \left(B_{1}^{P} \times B_{2}^{P}\right)\left(\left(x_{1} x_{2}\right),\left(y_{1} y_{2}\right)\right)=T\left(B_{1}^{P}\left(x_{1} y_{1}\right), B_{2}^{P}\left(x_{2} y_{2}\right)\right) \\
\leq & \left(\frac{A_{1}^{P}\left(x_{1}\right) A_{1}^{P}\left(y_{1}\right)}{A_{1}^{P}\left(x_{1}\right)+A_{1}^{P}\left(y_{1}\right)-A_{1}^{P}\left(x_{1}\right) A_{1}^{P}\left(y_{1}\right)},\right. \\
& \left.\frac{A_{2}^{P}\left(x_{2}\right) A_{2}^{P}\left(y_{2}\right)}{A_{2}^{P}\left(x_{2}\right)+A_{2}^{P}\left(y_{2}\right)-A_{2}^{P}\left(x_{2}\right) A_{2}^{P}\left(y_{2}\right)}\right)
\end{aligned}
$$

By putting $a=\eta_{1}\left(x_{1}\right), b=\eta_{1}\left(y_{1}\right), c=\eta_{2}\left(x_{2}\right), d=\eta_{2}\left(y_{2}\right)$, we get

$$
\begin{aligned}
\left(B_{1}^{P} \times B_{2}^{P}\right) & \left(\left(x_{1} x_{2}\right),\left(y_{1} y_{2}\right)\right) \leq T\left(\frac{a b}{a+b-a b}, \frac{c d}{c+d-c d}\right) \\
= & \frac{\frac{a b}{a+b-a b}+\frac{a d d}{c+d-c d}-\frac{a b c}{(a+b-a b+d-c d)}}{(a b c b d)} \\
= & \frac{\frac{a c}{(a+c-a c b)(b+d-b d)}}{\frac{a c+d-c d)}{a+c-a c}+\frac{b d}{b+d-b d}-\frac{a c b d}{(a+c-a c)(b+d-b d)}} \\
= & {\left[\left(A_{1} \times A_{2}\right)\left(\left(x_{1}, x_{2}\right)\right)\left(A_{1} \times A_{2}\right)\left(\left(y_{1}, y_{2}\right)\right)\right] } \\
& /\left[\left(A_{1} \times A_{2}\right) P\left(\left(x_{1}, x_{2}\right)\right)+\left(A_{1} \times A_{2}\right)^{P}\left(\left(y_{1}, y_{2}\right)\right)\right. \\
& \left.-\left(A_{1} \times A_{2}\right)^{P}\left(\left(x_{1}, x_{2}\right)\right)\left(A_{1} \times A_{2}\right)^{P}\left(\left(y_{1}, y_{2}\right)\right)\right]
\end{aligned}
$$

Similarly

$$
\begin{aligned}
& \left(B_{1}^{N} \times B_{2}^{N}\right)\left(\left(x_{1} x_{2}\right),\left(y_{1} y_{2}\right)\right) \leq T\left(\frac{a b}{a+b-a b}, \frac{c d}{c+d-c d}\right) \\
& =\frac{\frac{a b c d}{(a+b-a b)(c+d-c d)}}{\frac{a b}{a+b-a b}+\frac{c d}{c+d-c d}+\frac{a b c d}{(a+b-a b)(c+d-c d)}} \\
& =\frac{\frac{a c b d}{(a+c-a c)(b+d-b d)}}{\frac{a c}{a+c-a c}+\frac{b d}{b+d-b d}+\frac{a c b d}{(a+c-a c)(b+d-b d)}} \\
& =\left[\left(A_{1} \times A_{2}\right)^{N}\left(\left(x_{1}, x_{2}\right)\right)\left(A_{1} \times A_{2}\right)^{N}\left(\left(y_{1}, y_{2}\right)\right)\right] \\
& /\left[\left(A_{1} \times A_{2}\right)^{N}\left(\left(x_{1}, x_{2}\right)\right)+\left(A_{1} \times A_{2}\right)^{N}\left(\left(y_{1}, y_{2}\right)\right)\right. \\
& \text { - } \left.\left(A_{1} \times A_{2}\right)^{N}\left(\left(x_{1}, x_{2}\right)\right)\left(A_{1} \times A_{2}\right)^{N}\left(\left(y_{1}, y_{2}\right)\right)\right]
\end{aligned}
$$

Hence the direct product $G_{1} \times G_{2}$ is the dombi bipolar fuzzy graph.

Definition 2.4: Let $A_{i}$ be a bipolar fuzzy subset of $V_{i}$ and let $B_{i}$ be a fuzzy subset of $E_{i}, i=1,2, \ldots$ Define the Cartesian product $G_{1} \square G_{2}=\left(A_{1} \square A_{2}, B_{1} \square B_{2}\right)$ of dombi bipolar fuzzy graph $G_{1}=\left(A_{1}, B_{2}\right)$ and $G_{2}=\left(A_{2}, B_{2}\right)$ of $\left(V_{1}, E_{1}\right)$ and $\left(V_{2}, E_{2}\right)$, respectively,

(i) $\left(A_{1} \square A_{2}\right)^{P}\left(x_{1} x_{2}\right) \quad=\quad \frac{A_{1}^{P}\left(x_{1}\right) A_{2}^{P}\left(x_{2}\right)}{A_{1}^{P}\left(x_{1}\right)+A_{2}^{P}\left(x_{2}\right)-A_{1}^{P}\left(x_{1}\right) A_{2}^{P}\left(x_{2}\right)}$ for all $\left(x_{1}, x_{2}\right) \in V_{1} \times V_{2}$

(ii) $\left(A_{1} \square A_{2}\right)^{N}\left(x_{1} x_{2}\right)=\frac{A_{1}^{N}\left(x_{1}\right) A_{2}^{N}\left(x_{2}\right)}{A_{1}^{N}\left(x_{1}\right)+A_{2}^{N}\left(x_{2}\right)-A_{1}^{N}\left(x_{1}\right) A_{2}^{N}\left(x_{2}\right)}$ for all $\left(x_{1}, x_{2}\right) \in V_{1} \times V_{2}$

(iii)

$$
\left(B_{1} \square B_{2}\right)^{P}\left(\left(x x_{2}\right)\left(x y_{2}\right)\right) \quad=
$$
$\frac{A_{1}^{P}(x) B_{2}^{P}\left(x_{2} y_{2}\right)}{A_{1}^{P}(x)+B_{2}^{P}\left(x_{2} y_{2}\right)-A_{1}^{P}(x) B_{2}^{P}\left(x_{2} y_{2}\right)}$ for all $x \in V_{1}, x_{2} y_{2} \in E_{2}$

(iv) $\quad\left(B_{1} \square B_{2}\right)^{N}\left(\left(x x_{2}\right)\left(x y_{2}\right)\right)=$ $\frac{A_{1}^{N}(x) B_{2}^{N}\left(x_{2} y_{2}\right)}{A_{1}^{N}(x)+B_{2}^{N}\left(x_{2} y_{2}\right)-A_{1}^{N}(x) B_{2}^{N}\left(x_{2} y_{2}\right)}$ for all $x \in V_{1}, x_{2} y_{2} \in E_{2}$ $\left(B_{1} \square B_{2}\right)^{P}\left(\left(x_{1} z\right)\left(y_{1} z\right)\right) \quad=$ $\frac{A_{2}^{P}(z) B_{1}^{P}\left(x_{1} y_{1}\right)}{A_{2}^{P}(z)+B_{1}^{P}\left(x_{1} y_{1}\right)-A_{2}^{P}(z) B_{1}^{P}\left(x_{1} y_{1}\right)}$ for all $x_{1}, y_{1} \in E_{1} z \in E_{2}$
(vi) $\left(B_{1} \square B_{2}\right)^{N}\left(\left(x_{1} z\right)\left(y_{1} z\right)\right)$ $\frac{A_{2}^{N}(z) B_{1}^{N}\left(x_{1} y_{1}\right)}{A_{2}^{N}(z)+B_{1}^{N}\left(x_{1} y_{1}\right)-A_{2}^{N}(z) B_{1}^{N}\left(x_{1} y_{1}\right)}$ for all $z \in V_{2} x_{1} y_{1} \in E_{1}$

Remark 2.5: The Cartesian product of two bipolar dombi bipolar fuzzy graphs is not necessarily a dombi bipolar fuzzy graph.

Example 2.3: Consider two dombi fuzzy graphs $G_{1} \square G_{2}$. Then we have

$$
\begin{aligned}
\left(B_{1} \square B_{2}\right)((a c),(a d)) & =(0.25,-0.11) \\
\left(B_{1} \square B_{2}\right)((a d),(a e)) & =(0.20,-0.09) \\
\left(B_{1} \square B_{2}\right)((a e),(b e)) & =(0.2,-0.046) \\
\left(B_{1} \square B_{2}\right)((b e),(b d)) & =(0.2,-0.13) \\
\left(B_{1} \square B_{2}\right)((b d),(b c)) & =(0.18,-0.13) \\
\left(B_{1} \square B_{2}\right)((a d),(b d)) & =(0.2,-0.05) \\
\left(B_{1} \square B_{2}\right)((a c),(b c)) & =(0.2,-0.046) .
\end{aligned}
$$

Clearly $G_{1} \square G_{2}$ is not a dombi bipolar fuzzy graphs.

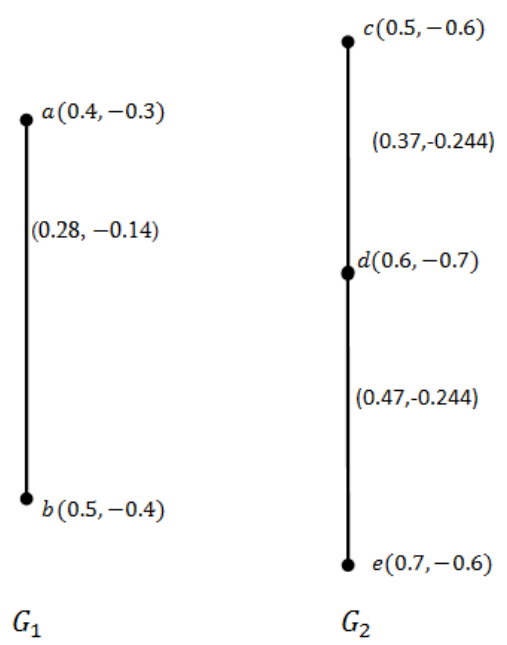

Definition 2.6: Let $A_{i}$ be a bipolar fuzzy subset of $V_{i}$ and let $B_{i}$ be a fuzzy subset of $E_{i}, i=1,2, \ldots$. Define the union 


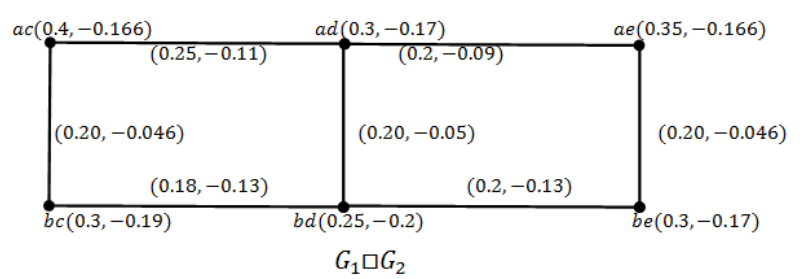

Figure 3 Cartesian product of dombi bipolar fuzzy graph

$G_{1} \cup G_{2}=\left(A_{1} \cup A_{2}, B_{1} \cup B_{2}\right)$ of dombi bipolar fuzzy graphs $G_{1}=\left(A_{1}, B_{1}\right)$ and $G_{2}=\left(A_{2}, B_{2}\right)$ be given as follows:

$$
\left(A_{1} \cup A_{2}\right)^{P}(x)=\left\{\begin{array}{l}
A_{1}^{P}(x) \quad \text { if } x \in V_{1} \backslash V_{2}, \\
A_{2}^{P}(x) \quad \text { if } x \in V_{2} \backslash V_{1}, \\
\frac{A_{1}^{P}(x) A_{1}^{P}(y)}{A_{1}^{P}(x)+A_{1}^{P}(y)-A_{1}^{P}(x) A_{1}^{P}(y)} \text { if } x \in V_{1} \cap V_{2} .
\end{array}\right.
$$$$
\left(A_{1} \cup A_{2}\right)^{N}(x)=\left\{\begin{array}{l}
A_{1}^{N}(x) \quad \text { if } x \in V_{1} \backslash V_{2}, \\
A_{2}^{N}(x) \quad \text { if } x \in V_{2} \backslash V_{1}, \\
\frac{A_{1}^{N}(x) A_{1}^{N}(y)}{A_{1}^{N}(x)+A_{1}^{N}(y)-A_{1}^{N}(x) A_{1}^{N}(y)} \text { if } x \in V_{1} \cap V_{2} .
\end{array}\right.
$$

Also,

$$
\begin{gathered}
\left(B_{1} \cup B_{2}\right)^{P}(x y)=\left\{\begin{array}{lr}
B_{1}^{P}(x y) / 2 & \text { if } x y \in E_{1} \backslash E_{2}, \\
B_{2}^{P}(x y) / 2 & \text { if } x y \in E_{2} \backslash E_{1}, \\
\frac{A_{1}^{P}(x) A_{1}^{P}(y)}{A_{1}^{P}(x)+A_{1}^{P}(y)-A_{1}^{P}(x) A_{1}^{P}(y)} / 2
\end{array}\right. \\
\left(B_{1} \cup B_{2}\right)^{N}(x y)= \begin{cases}B_{1}^{N}(x y) / 2 & \text { if } x y \in E_{1} \cap E_{2} . \\
B_{2}^{N}(x y) / 2 & \text { if } x y \in E_{1} \backslash E_{2}, \\
\frac{A_{1}^{N}(x) A_{1}^{N}(y)}{A_{1}^{N}(x)+A_{1}^{N}(y)-A_{1}^{N}(x) A_{1}^{N}(y)} / 2 & \text { if } x y \in E_{1} \cap E_{2} .\end{cases}
\end{gathered}
$$

Example 2.4: The dombi fuzzy graphs are, $G_{1}=\{j(0.5,-0.5), k(0.8,-0.8), l(0.4,-0.4)\} \quad$ and $G_{2}=\{j(0.6,-0.6), k(0.5,-0.5), m(0.3,-0.3)\}$. Then we have $A_{1} \cup A_{2}=\frac{j}{(0.37,-0.21)}, \frac{k}{(0.4,-0.23)}, \frac{l}{(0.4,-0.4)}, \frac{m}{(0.3,-0.3)}$ $B_{1} \cup B_{2}=\frac{j l}{(0.05,-0.09)}, \frac{j k}{(0.11,-0.09)}, \frac{m k}{(0.05,-0.075)}, \frac{l k}{(0.15,-0.1)}$

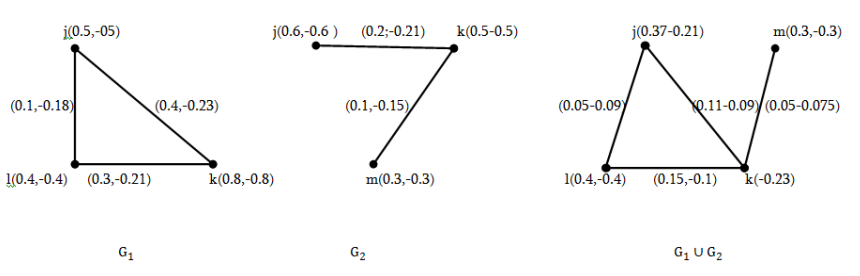

Figure 4 Union of dombi bipolar fuzzy graph

Theorem 2.7: The union $G_{1} \cup G_{2}$ of $G_{1}$ and $G_{2}$ is the dombi bipolar fuzzy graph of $G$ if and only if $G_{1}$ and $G_{2}$ are the dombi bipolar fuzzy graphs of $G$, where $A_{1}, A_{2}, B_{1}$ and $B_{2}$ are bipolar subsets of $V_{1}, V_{2}, E_{1}$ and $E_{2}$ respectively, provided $V_{1} \cap V_{2}=\phi$.
Proof: Assume that $G_{2} \cup G_{2}$ are dombi bipolar fuzzy graphs. Let $x y \in E_{1}$, then $x y \in E_{2}$, and $x, y \in V_{1} \backslash V_{2}$. Then

$$
\begin{aligned}
& B_{1}^{P}(x y)=\left(B_{1} \cup B_{2}\right)^{P}(x y) \\
& \leq\left[\left(A_{1}^{P} \cup A_{2}^{P}\right)(x)\left(A_{1}^{P} \cup A_{2}^{P}\right)(y)\right] /\left[\left(A_{1}^{P} \cup A_{2}^{P}\right)(x)\right. \\
& \left.+\left(A_{1}^{P} \cup A_{2}^{P}\right)(y)-\left(A_{1}^{P} \cup A_{2}^{P}\right)(x)\left(A_{1}^{P} \cup A_{2}^{P}\right)(y)\right] \\
& B_{1}^{P}(x y) \leq \frac{A_{1}^{P}(x) A_{1}^{P}(y)}{A_{1}^{P}(x)+A_{1}^{P}(y)-A_{1}^{P}(x) A_{1}^{P}(y)}
\end{aligned}
$$

Thus $G_{1}$ is the dombi bipolar fuzzy graph of $G$. Similarly it is easy to verify that $G_{2}$ is the dombi bipolar fuzzy graph of $G$. Conversely, assume that $G_{1}$ and $G_{2}$ are the dombi bipolar fuzzy graph of $G$ respectively, consider $x y \in E_{1} \backslash E_{2}$ then by the definition of union, it follows that,

$$
\begin{aligned}
\left(B_{1} \cup B_{2}\right)^{P}(x y) & =B_{1}^{P}(x y) \leq\left(A_{1}^{P}(x), A_{1}^{P}(y)\right) \\
= & \left(\left(A_{1} \cup A_{2}\right)^{P}(x),\left(A_{1} \cup A_{2}\right)^{P}(x)\right) .
\end{aligned}
$$

In the similar way, we can find $x y \in E_{2} \backslash E_{1}$,

$$
\begin{aligned}
& \left(B_{1} \cup B_{2}\right)^{P}(x y) \leq\left(\left(A_{1} \cup A_{2}\right)(x),\left(A_{1} \cup A_{2}\right)(x)\right) \\
\leq & {\left[\left(A_{1} \cup A_{2}\right)^{P}(x)\left(A_{1} \cup A_{2}\right)^{P}(y)\right] /\left[\left(A_{1} \cup A_{2}\right)^{P}(x)\right.} \\
& \left.+\left(A_{1} \cup A_{2}\right)^{P}(y)-\left(A_{1} \cup A_{2}\right)^{P}(x)\left(A_{1} \cup A_{2}\right)^{P}(y)\right] .
\end{aligned}
$$

Also,

$$
\begin{aligned}
& B_{1}^{N}(x y) \leq \frac{A_{1}^{N}(x) A_{1}^{N}(y)}{A_{1}^{N}(x)+A_{1}^{N}(y)-A_{1}^{N}(x) A_{1}^{N}(y)} \\
& \left(B_{1} \cup B_{2}\right)^{N} \leq\left[\left(A_{1} \cup A_{2}\right)^{N}(x)\left(A_{1} \cup A_{2}\right)^{N}(y)\right] /\left[\left(A_{1} \cup A_{2}\right)^{N}(x)\right. \\
& \left.+\left(A_{1} \cup A_{2}\right)^{N}(y)-\left(A_{1} \cup A_{2}\right)^{N}(x)\left(A_{1} \cup A_{2}\right)^{N}(y)\right] .
\end{aligned}
$$

Definition 2.8: Let $A_{i}$ be a bipolar fuzzy subset of $V_{i}$ and let $B_{i}$ be a bipolar fuzzy subset of $E_{i}, i=1,2 \ldots$ Define the ring sum $G_{1} \oplus G_{2}=\left(A_{1} \oplus A_{2}, B_{1} \oplus B_{2}\right)$ of the dombi bipolar fuzzy graphs, where $G_{1}=\left(A_{1}, B_{1}\right)$ and $G_{2}=\left(A_{2}, B_{2}\right)$ satisfies the following conditions:

(i)

$$
\left(A_{1} \oplus A_{2}\right)^{P}(x)=\left(A_{1} \cup A_{2}\right)^{P}(x) \text { if } x \in V_{1} \cup V_{2}
$$

$$
\left(A_{1} \oplus A_{2}\right)^{N}(x)=\left(A_{1} \cup A_{2}\right)^{N}(x) \text { if } x \in V_{1} \cup V_{2}
$$

$$
\left(B_{1} \oplus B_{2}\right)^{P}(x y)= \begin{cases}B_{1}^{P}(x y), & \text { if } x y \in E_{1} \backslash E_{2}, \\ B_{2}^{P}(x y), & \text { if } x y \in E_{2} \backslash E_{1}, \\ 0, & \text { if } x y \in E_{1} \cap E_{2} .\end{cases}
$$

(iv)

$$
\left(B_{1} \oplus B_{2}\right)^{N}(x y)= \begin{cases}B_{1}^{N}(x y), & \text { if } x y \in E_{1} \backslash E_{2}, \\ B_{2}^{N}(x y), & \text { if } x y \in E_{2} \backslash E_{1}, \\ 0, & \text { if } x y \in E_{1} \cap E_{2} .\end{cases}
$$

Proposition 2.9: The ring sum $G_{1} \oplus G_{2}$ of two bipolar dombi fuzzy graphs $G_{1}$ and $G_{2}$ of $G_{1}^{*}$ and $G_{2}^{*}$ is the dombi bipolar fuzzy graph of $G_{1} \oplus G_{2}$. 
Proof: Consider $x y \in E_{1} \backslash E_{2}$, then there are three possibilities such as:

(i) $x, y \in V_{1} \backslash V_{2}$,

(ii) $x \in V_{1} \backslash V_{2}, y \in V_{1} \cap V_{2}$,

(iii) $x, y \in V_{1} \cap V_{2}$.

(i) Suppose $x, y \in V_{1} \backslash V_{2}$, then by the Definition of ring sum we have,

$$
\begin{aligned}
\left(B_{1} \oplus B_{2}\right)^{P}(x y) & =B_{1}^{P}(x y) \\
\leq & \left(A_{1}^{P}(x), A_{1}^{P}(y)\right) \\
\leq & \left(\left(A_{1} \cup A_{2}\right)^{P}(x),\left(A_{1} \cup A_{2}\right)^{P}(y)\right) \\
\leq & \left(\left(A_{1} \oplus A_{2}\right)^{P}(x),\left(A_{1} \oplus A_{2}\right)^{P}(y)\right)
\end{aligned}
$$

(ii) Suppose $x \in V_{1} \backslash V_{2}, y \in V_{1} \cap V_{2}$,

$$
\begin{aligned}
\left(B_{1} \oplus B_{2}\right)^{P}(x y) & =B_{1}^{P}(x y) \\
\leq & \left(A_{1}^{P}(x), A_{1}^{P}(y)\right) \\
\leq & \left(\left(A_{1} \cup A_{2}\right)^{P}(x), A_{1}^{P}(y)\right) \\
\leq & \left(\left(A_{1} \oplus A_{2}\right)^{P}(x), A_{1}^{P}(y)\right)
\end{aligned}
$$

Clearly,

$$
A_{1}(y) \leq \frac{A_{1}(y)+A_{2}(y)-2 A_{1}(y) A_{2}(y)}{1-A_{1}(y) A_{2}(y)} .
$$

By substituting, $A_{1}(y)=f, A_{2}(y)=h$, we have

$$
\begin{aligned}
f & \leq \frac{f+h-2 f h}{1-f h} \\
f-f^{2} h & \leq f+h-2 f h \\
0 & \leq(f-1)^{2} .
\end{aligned}
$$

Since $\left(B_{1} \oplus B_{2}\right)(x y) \leq\left(\left(A_{1} \oplus A_{2}\right)(x),\left(A_{1} \oplus A_{2}\right)(y)\right)$

(iii) Suppose $x, y \in V_{1} \cap V_{2}$. Then we get,

$$
\begin{aligned}
\left(B_{1} \oplus B_{2}\right)(x y) & =B_{1}(x y) \\
\leq & \left(A_{1}(x), A_{1}(y)\right) \\
\leq & \left(\left(A_{1} \cup A_{2}\right)(x),\left(A_{1} \cup A_{2}\right)(y)\right) \\
\leq & \left(\left(A_{1} \oplus A_{2}\right)^{P}(x),\left(A_{1} \oplus A_{2}\right)(y)\right)
\end{aligned}
$$

Since by (ii), we have

$$
\begin{aligned}
& A_{1}(x) \leq \frac{A_{1}(x)+A_{2}(x)-2 A_{1}(x) A_{2}(x)}{1-A_{1}(x) A_{2}(x)} \quad \text { and } \\
& A_{1}(y) \leq \frac{A_{1}(y)+A_{2}(y)-2 A_{1}(y) A_{2}(y)}{1-A_{1}(y) A_{2}(y)}
\end{aligned}
$$

Because of symmetry, we obtain for $x y \in E_{2} \backslash E_{1}$ in the three possible cases.

$$
\begin{aligned}
& \left(B_{1} \oplus B_{2}\right)^{P}(x y) \leq\left(\left(A_{1} \oplus A_{2}\right)^{P}(x),\left(A_{1} \oplus A_{2}\right)^{P}(y)\right) \\
\leq & {\left[\left(A_{1} \oplus A_{2}\right)^{P}(x)\left(A_{1} \oplus A_{2}\right)^{P}(y)\right] /\left[\left(A_{1} \oplus A_{2}\right)^{P}(x)\right.} \\
& \left.+\left(A_{1} \oplus A_{2}\right)^{P}(y)-\left(A_{1} \oplus A_{2}\right)^{P}(x)\left(A_{1} \oplus A_{2}\right)^{P}(y)\right]
\end{aligned}
$$

Hence proved.

Definition 2.10: The complement of D-Bipolar-fuzzy graphs $G=(A, B)$ of $G=(V, E)$ is a D-Bipolar-fuzzy graphs of

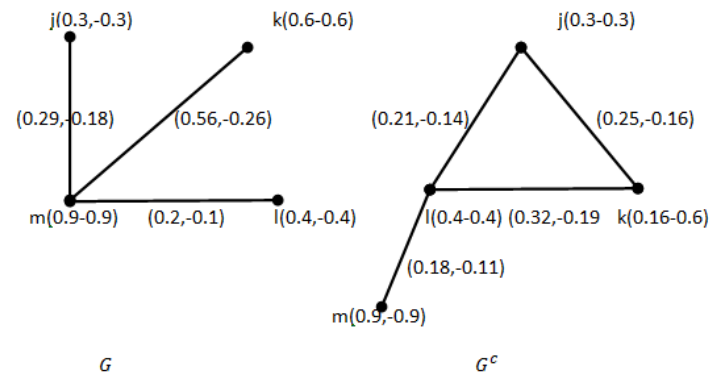

Figure 5 Complement of dombi bipolar fuzzy graph

$\bar{G}=(\bar{A}, \bar{B})$ where $\bar{A}(x)=A$ for all $x \in V$ and $\bar{B}$ is defined as follows.

$$
\bar{B}^{P}(x y)=\left\{\begin{array}{c}
\frac{A^{P}(x) A^{P}(y)}{A^{P}(x)+A^{P}(y)-A^{P}(x) A^{P}(y)}, \text { if } B^{P}(x y)=0 \\
\frac{A^{P}(x) A^{P}(y)}{A^{P}(x)+A^{P}(y)-A^{P}(x) A^{P}(y)}-A^{P}(x y), \\
\text { if } 0 \leq B^{P}(x y) \leq 1
\end{array}\right.
$$

$\bar{B}^{N}(x y)=\left\{\begin{array}{l}\frac{A^{N}(x) A^{N}(y)}{A^{N}(x)+A^{N}(y)-A^{N}(x) A^{N}(y)}, \text { if } B^{N}(x y)=0 \\ \frac{A^{N}(x) A^{N}(y)}{A^{N}(x)+A^{N}(y)-A^{N}(x) A^{N}(y)}-A^{N}(x y), \\ \text { if }-1 \leq B^{N}(x y) \leq 0\end{array}\right.$

Example 2.5: Consider a D-Bipolar-fuzzy graph over $V=$ $\{j, k, l, m\}$ defined by

$$
\begin{array}{r}
A=\left\{\frac{j}{0.3,-0.3}, \frac{k}{0.6,-0.6}, \frac{l}{0.4,-0.4}, \frac{m}{0.9,-0.9}\right\}, \\
B=\left\{\frac{j m}{0.29,-0.18}, \frac{k m}{0.56,0.26}, \frac{l m}{0.2,-0.1}\right\}
\end{array}
$$

we have

$$
\begin{aligned}
\overline{\overline{B^{P}(x y)}} & =\frac{\overline{A^{P}(x) A^{P}(y)}}{\overline{A^{P}(x)}+\overline{A^{P}(y)}+\overline{\overline{A^{P}(x) A^{P}(y)}}}-\overline{B^{P}(x y)} \\
= & \frac{A^{P}(x) A^{P}(y)}{A^{P}(x)+A^{P}(y)-A^{P}(x) A^{P}(y)} \\
& -\left(\frac{A^{P}(x) A^{P}(y)}{A^{P}(x)+A^{P}(y)-A^{P}(x) A^{P}(y)}-B^{P}(x y)\right) \\
= & B^{P}(x y) \text { for every } x, y \in V .
\end{aligned}
$$

Hence $\overline{\bar{G}}=G$

Definition 2.11: A homomorphism $\chi:\left(G_{1} \rightarrow G_{2}\right)$ of two D-Bipolar-fuzzy graphs $G_{1}=\left(A_{1}, B_{1}\right)$ and $G_{2}=\left(A_{2}, B_{2}\right)$ is a mapping $\chi: V_{1} \rightarrow V_{2}$ satisfying the following condition:

(i) $A_{1}^{P}(x) \leq A_{2}^{P}(\chi(x))$ for all $x \in V_{1}$

(ii) $A_{1}^{N}(x) \geq A_{2}^{N}(\chi(x))$ for all $x \in V_{1}$

(ii) $B_{1}^{P}(x y) \leq B_{2}^{P}(\chi(x y))$ for all $x y \in V_{2}$

(iv) $B_{1}^{N}(x y) \geq B_{2}^{N}(\chi(x y))$ for all $x y \in V_{2}$

Definition 2.12: A isomorphism $\chi:\left(G_{1} \rightarrow G_{2}\right)$ of two D-Bipolar-fuzzy graphs $G_{1}=\left(A_{1}, B_{1}\right)$ and $G_{2}=$ $\left(A_{2}, B_{2}\right)$ (denoted as $\left.G_{1} \cong G_{2}\right)$ is a bijective mapping $\chi$ : $V_{1} \rightarrow V_{2}$ satisfying the following condition:

(v) $A_{1}^{P}(x)=A_{2}^{P}(\chi(x))$ for all $x \in V_{1}$

(vi) $A_{1}^{N}(x)=A_{2}^{N}(\chi(x))$ for all $x \in V_{1}$ 
(vii) $B_{1}^{P}(x y)=B_{2}^{P}(\chi(x y))$ for all $x y \in V_{2}$

(viii) $B_{1}^{N}(x y)=B_{2}^{N}(\chi(x y))$ for all $x y \in V_{2}$

A weak isomorphism $\chi:\left(G_{1} \rightarrow G_{2}\right)$ is a bijective homomorphism with the condition (v) and (vi) above and a co-weak isomorphism $\chi:\left(G_{1} \rightarrow G_{2}\right)$ is a bijective homomorphism with the condition (vii) and (viii) above

Definition 2.13: A D-Bipolar-fuzzy graph $G=(A, B)$ is said to be Self-complementary if $G=(A, B) \cong \bar{G}=(\bar{A}, \bar{B})$.

Proposition 2.14: Let $G=(A, B)$ be a self complementary D-Bipolar-fuzzy graph, then

$$
\begin{gathered}
\sum_{x \neq y} B^{P}(x y)=\frac{1}{2} \sum_{x \neq y} \frac{A^{P}(x) A^{P}(y)}{A^{P}(x)+A^{P}(y)-A^{P}(x) A^{P}(y)} \text { and } \\
\sum_{x \neq y} B^{N}(x y)=\frac{1}{2} \sum_{x \neq y} \frac{A^{N}(x) A^{N}(y)}{A^{N}(x)+A^{N}(y)-A^{N}(x) A^{N}(y)}
\end{gathered}
$$

Proof: Let $G$ be a self-complementary D-Bipolar-fuzzy graph. then there exists an isomorphism $\chi: V \rightarrow V$ such that $\overline{A^{P}}(\chi(x))=A^{P}(x)$ for all $x \in V$ and $\overline{A^{P}}(\chi(x) \chi(y))=$ $A^{P}(x y)$ for all $x y \in E$. By definition of $\bar{G}$, we have

$$
\begin{aligned}
\overline{B^{P}}(\chi(x) \chi(y)) & =\frac{\overline{A^{P}} \chi(x) \overline{A^{P}} \chi(y)}{\overline{A^{P}} \chi(x)+\overline{A^{P}} \chi(y)+\overline{A^{P}} \chi(x) \overline{A^{P}} \chi(y)} \\
& -B^{P}(\chi(x) \chi(y)) \\
B^{P}(x y) & =\frac{A^{P}(x) A^{P}(y)}{A^{P}(x)+A^{P}(y)-A^{P}(x) A^{P}(y)} \\
& -B^{P}(\chi(x) \chi(y)) \\
\sum_{x \neq y} B^{P}(x y) & =\frac{A^{P}(x) A^{P}(y)}{A^{P}(x)+A^{P}(y)-A^{P}(x) A^{P}(y)} \\
& -B^{P}(\chi(x) \chi(y)) \\
\sum_{x \neq y} B^{P}(x y) & +\sum_{x \neq y} B^{P}(\chi(x) \chi(y)) \\
& =\sum_{x \neq y} \frac{A^{P}(x) A^{P}(y)}{A^{P}(x)+A^{P}(y)-A^{P}(x) A^{P}(y)} \\
2 \sum_{x \neq y} B^{P}(x y) & =\sum_{x \neq y} \frac{A^{P}(x) A^{P}(y)}{A^{P}(x)+A^{P}(y)-A^{P}(x) A^{P}(y)} \\
\sum_{x \neq y} B^{P}(x y) & =\frac{1}{2} \sum_{x \neq y} \frac{A^{P}(x) A^{P}(y)}{A^{P}(x)+A^{P}(y)-A^{P}(x) A^{P}(y)}
\end{aligned}
$$

Similarly we prove that

$$
\sum_{x \neq y} B^{N}(x y)=\frac{1}{2} \sum_{x \neq y} \frac{A^{N}(x) A^{N}(y)}{A^{N}(x)+A^{N}(y)-A^{N}(x) A^{N}(y)} \text {. Hence }
$$
proved.

Proposition 2.15: Let $G=(S, A)$ be the D-Bipolar-fuzzy graph of $G_{*}$.

If $B^{P}(x y)=\frac{1}{2} \frac{A^{P}(x) A^{P}(y)}{A^{P}(x)+A^{P}(y)-A^{P}(x) A^{P}(y)}$ and $B^{N}(x y)=$ $\frac{1}{2} \frac{A^{N}(x) A^{N}(y)}{A^{N}(x)+A^{N}(y)-A^{N}(x) A^{N}(y)}$ for all $x, y \in V$, then $G$ is selfcomplementary.

Proof: Let $G$ be the D-Bipolar-fuzzy graph satisfying $B^{P}(x y)=\frac{1}{2} \frac{A^{P}(x) A^{P}(y)}{A^{P}(x)+A^{P}(y)-A^{P}(x) A^{P}(y)}$ for all $x, y \in V$, then the identity mapping $I: V \rightarrow V$ is an isomorphism from $G$ to
$\bar{G}$. Clearly, $I$ satisfies the condition $(v)$ and $(v i)$ of definition 3.13. Since $B^{P}(x y)=\frac{1}{2} \frac{A^{P}(x) A^{P}(y)}{A^{P}(x)+A^{P}(y)-A^{P}(x) A^{P}(y)}$, we have

$$
\begin{aligned}
\overline{B^{P}}(I(x) & I(y))=\bar{B}(x y) \\
& =\frac{A^{P}(x) A^{P}(y)}{A^{P}(x)+A^{P}(y)-A^{P}(x) A^{P}(y)}-B^{P}(x y) \\
& =\frac{A^{P}(x) A^{P}(y)}{A^{P}(x)+A^{P}(y)-A^{P}(x) A^{P}(y)} \\
& -\frac{1}{2}\left(\frac{A^{P}(x) A^{P}(y)}{A^{P}(x)+A^{P}(y)-A^{P}(x) S(y)}\right) \\
& =\frac{1}{2}\left(\frac{A^{P}(x) A^{P}(y)}{A^{P}(x)+A^{P}(y)-A^{P}(x) A^{P}(y)}\right)=B^{P}(x y)
\end{aligned}
$$

Thus the condition (vii) and (viii) of Definition 3.13 is also satisfied by $I$ therefore $G$ is self complementary.

Proposition 2.16: The complements of two isomorphic DBipolar-fuzzy graphs are isomorphic and conversely.

Proof: Suppose that $G_{1}$ and $G_{2}$ are two isomorphic D-Bipolargraphs. then there exists a bijective mapping $\chi: V_{1} \rightarrow V_{2}$ satisfying

(i) $A_{1}^{P}(x)=A_{2}^{P}(\chi(x))$ for all $x \in V_{1}$

(ii) $A_{1}^{N}(x)=A_{2}^{N}(\chi(x))$ for all $x \in V_{1}$

(iii) $B_{1}^{P}(x y)=B_{2}^{P}(\chi(x y))$ for all $x y \in V_{2}$

(iv) $B_{1}^{N}(x y)=B_{2}^{N}(\chi(x y))$ for all $x y \in V_{2}$

using the definition of complement, we have

$$
\begin{aligned}
\overline{B^{P}}{ }_{1}(x y) & =\frac{A_{1}^{P}(x) A_{1}^{P}(y)}{A_{1}^{P}(x)+A_{1}^{P}(y)-A_{1}^{P}(x) A_{1}^{P}(y)}-B_{1}^{P}(x y) \\
& =\frac{S_{2}(\chi(x)) A_{2}^{P}(\chi(y))}{A_{2}^{P}(\chi(x))+\left(A_{2}^{P} \chi(y)\right)-\left(A_{2}^{P} \chi(x)\right)\left(A_{2}^{P} \chi(y)\right)} \\
& -B_{2}^{P}(\chi(x) \chi(y))=\overline{B_{2}^{P}}(\chi(x) \chi(y)) .
\end{aligned}
$$

hence $\overline{G_{1}} \cong \overline{G_{2}}$. Similarly, we can prove the converse part.

\section{STRONG D-BIPOLAR-FUZZY GRAPH}

Definition 3.1: A strong D-Bipolar-fuzzy graph $G=(A, B)$ is called a strong D-N-fuzzy graph of $G=(V, E)$ if

$$
B^{P}(x y)=\frac{A^{P}(x) A^{P}(y)}{A^{P}(x)+A^{P}(y)-A^{P}(x) A^{P}(y)}
$$

and

$$
B^{N}(x y)=\frac{A^{N}(x) A^{N}(y)}{A^{N}(x)+A^{N}(y)-A^{N}(x) A^{N}(y)}
$$

for all $x y \in E$

Example 3.1: Consider a D-Bipolar-fuzzy graph over $V=$ $a, b, c, d$ defined by

$$
\begin{gathered}
A=\left\{\frac{j}{-0.6}, \frac{k}{-0.7}, \frac{l}{-0.9}, \frac{m}{-0.4}\right\} \\
B=\left\{\frac{j k}{-0.47}, \frac{j m}{-0.3}, \frac{l m}{-0.38}\right\} .
\end{gathered}
$$




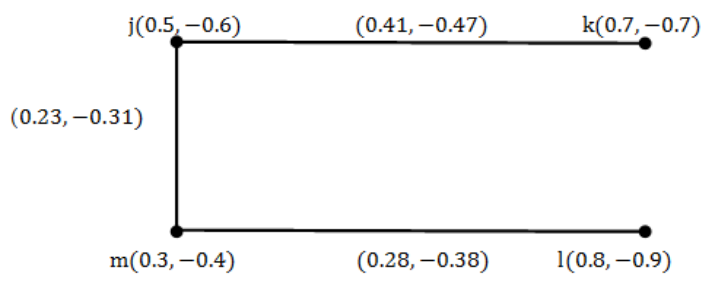

G

Figure 6 Strong dombi bipolar fuzzy graph

Definition 3.2: A D-Bipolar-fuzzy graph $G=(A, B)$ is said to be a complete D-Bipolar-fuzzy graph of $G^{*}=(V, E)$ if

$$
B^{P}(x y)=\frac{A^{P}(x) A^{P}(y)}{A^{P}(x)+A^{P}(y)-A^{P}(x) A^{P}(y)}
$$

and

$$
B^{N}(x y)=\frac{A^{N}(x) A^{N}(y)}{A^{N}(x)+A^{N}(y)-A^{N}(x) A^{N}(y)}
$$

for all $x y \in V$

Remark 3.3: Every complete D-Bipolar-fuzzy graph is strong D-Bipolar-fuzzy graph.

\section{REFERENCES}

[1] Akram, M. Bipolar fuzzy graphs. Information sciences, 181(24) (2011), 5548-5564.

[2] Akram, M. Bipolar fuzzy graphs with applications. Knowledge-Based Systems, 39 (2013), 1-8.

[3] Akram, M., Dudek, W. A. Regular bipolar fuzzy graphs. Neural Computing and Applications, 21(1) (2012), 197-205.

[4] Ashraf, S., Naz, S., Kerre, E. E. Dombi Fuzzy Graphs. Fuzzy Information and Engineering, 10(1)(2018), 58-79.

[5] Dombi, J. A general class of fuzzy operators, the De Morgan class of fuzzy operators and fuzziness measures induced by fuzzy operators. Fuzzy sets and systems, 8(2)(1982), 149-163.

[6] R. Jahir Hussain, S. Satham Hussain Triple Connected Domination Number of a Bipolar Fuzzy Graph, International Journal For Research in Applied Science and Engineering Technology, 5(8), 2017. DOI: 10.22214/ijraset.2017.8001

[7] Rashmanlou, H., Samanta, S., Pal, M., Borzooei, R. A. Product of bipolar fuzzy graphs and their degree. International Journal of General Systems, 45(1) (2016), 1-14.

[8] Rashmanlou, H., Samanta, S., Pal, M., Borzooei, R. A. A study on bipolar fuzzy graphs. Journal of Intelligent and Fuzzy Systems, 28(2) (2015), 571-580.

[9] Sarwar, M., Akram, M. Novel concepts of bipolar fuzzy competition graphs. Journal of Applied Mathematics and Computing, 54 (2017), 511547.

[10] Ullah, K., Mahmood, T., Jan, N., Broumi, S., Khan, Q. On bipolar-valued hesitant fuzzy sets and their applications in multi-attribute decision making. The Nucleus, 55(2) (2018), 93-101.

[11] W. R. Zhang, Bipolar fuzzy sets and relations: a computational framework for cognitive modeling and multiagent decision analysis, Proceedings of IEEE Conf., 1994, 305309.

[12] W. R. Zhang, Bipolar fuzzy sets, Proceedings of FUZZ-IEEE (1998), 835840 . 